\title{
El reto del envejecimiento
}

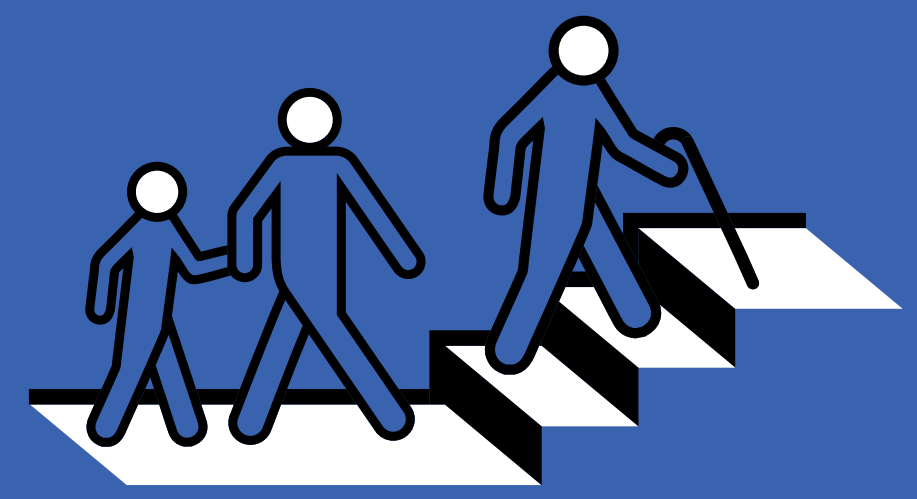

\section{El programa SABIEX de la UMH impulsa la investigación para promover la salud en mayores de 55 años}

\section{Belén Pardos}

no de los principales retos de las sociedades actuales pasa por ofrecer unas condiciones de vida adecuadas a las necesidades de las personas mayores. La longevidad del ser humano supone un triunfo evolutivo, pero también conlleva desafios relacionados con las particularidades de esta etapa. El programa SABIEX de la Universidad Miguel Hernández (UMH) de Elche impulsa la investigación desde diferentes áreas de conocimiento para ofrecer soluciones integrales destinadas a promover el envejecimiento activo y saludable.

La directora de SABIEX, Esther Sitges, explica que el programa se fundamenta en el compromiso de los investigadores para optimizar la calidad de vida de las personas mayores, a través de la formación y la investigación. Desde SABIEX se promueven investigaciones relacionadas, por ejemplo, con la actividad física, la participación social o la nutrición, encaminadas a fomentar su bienestar. La vicerrectora adjunta del Vicerrectorado de Cultura y Extensión Universitaria de la UMH del que depende

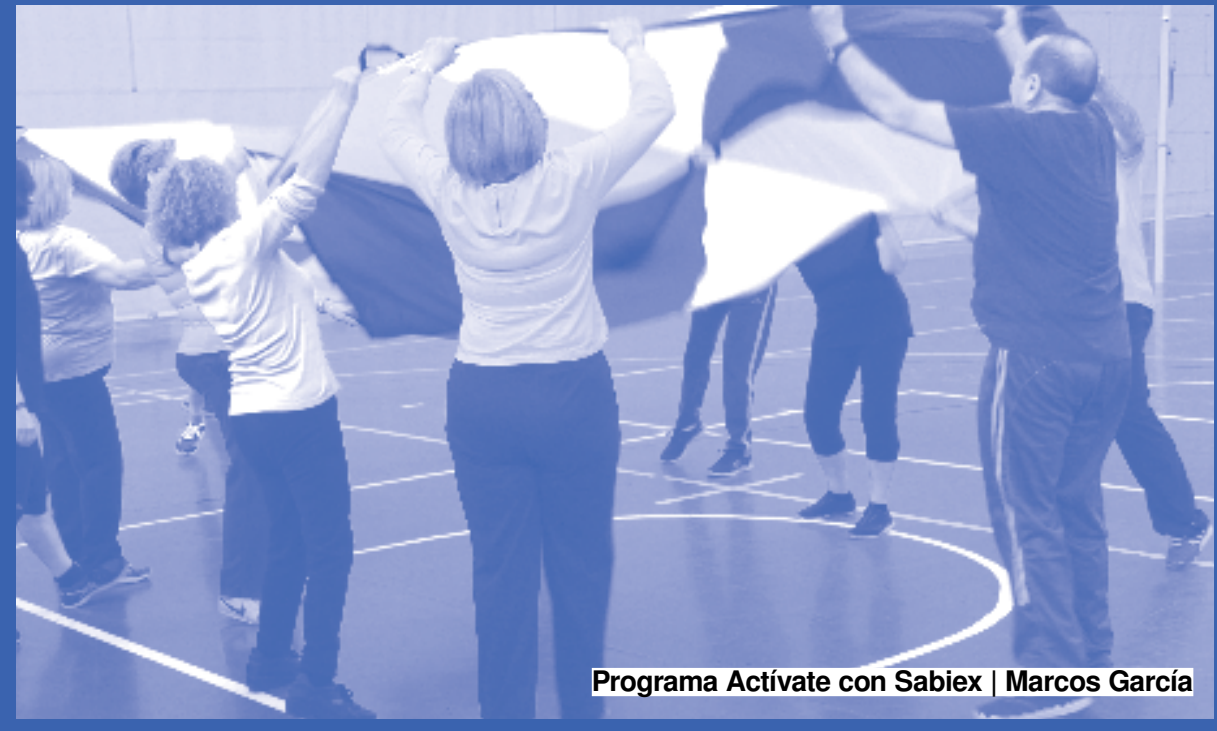

SABIEX, Esther Fuentes, explica que el programa engloba diversos estudios, ya que esta línea de trabajo en torno a la mejora de la calidad de vida de los mayores se desarrolla desde hace más de diez años.

Las personas de edad avanzada son quienes más recursos sanitarios necesitan $y$, por tanto, generan mayor gasto sanitario Los datos de múltiples estudios demuestran que se puede mejorar su salud a través de diferentes acciones. En este sentido, las dos investigadoras de SABIEX subrayan que la situación requiere centrar esfuerzos para promover la salud a lo largo del ciclo vital. 


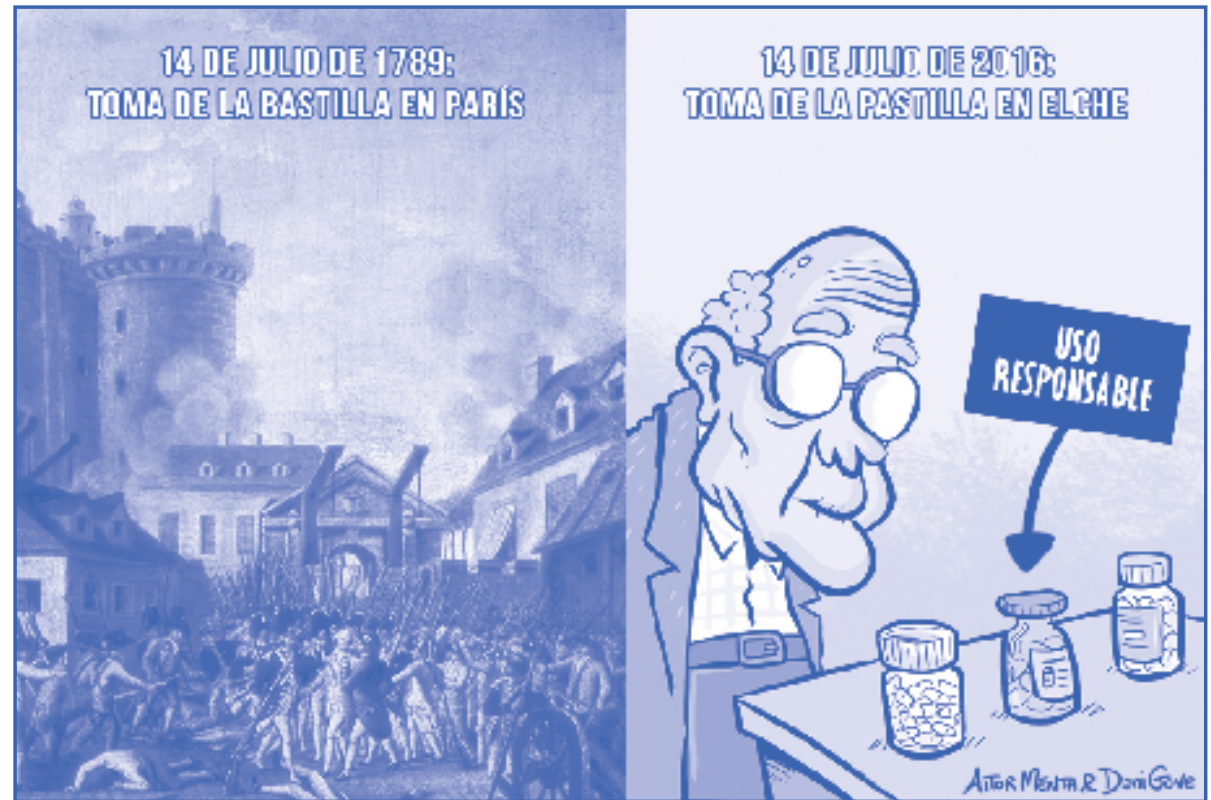

$>$ Las personas que participan en los programas universitarios de formación para mayores que ofrece SABIEX también colaboran en los diferentes estudios. De esta manera, los investigadores pueden analizar cuestiones relacionadas con las necesidades de este tramo poblacional y, a su vez, los mayores se benefician de programas de actividad física, de nutrición o de estimulación cognitiva.

\section{Ejercicio físico y actividad cerebral}

Una de las investigaciones actuales incluye el programa 'Actívate con SABIEX', que pretende profundizar en la relación entre actividad física y cognición. 'Actívate' se articula en dos propuestas complementarias, 'Fisiogym' y 'Neurogym', coordinadas por los profesores de la UMH Diego Pastor y Beatriz Bonete, respectivamente. La literatura científica recoge numerosas evidencias que relacionan la actividad física con un efecto positivo del rendimiento en la cognición de los mayores. Por este motivo, los investigadores trabajan con el grupo de mayores de 55 años divididos en tres subgrupos. Por una parte, se encuentran los individuos que participan en las dos actividades, por otra, los que sólo realizan una de ellas y, también, quienes no forman parte de ninguna -el grupo control-.

Diego Pastor explica que 'Actívate' pretende valorar cómo cambia la cognición de los participantes en función de que realicen una actividad u otra, si son sumatorios los efectos y si es mejor uno que otro. La investigación se encuentra en una fase inicial que, una vez terminadas las mediciones, servirá para analizar los efectos agudo y crónico. Es decir, si el hecho de llevar a cabo una sesión de actividad física revierte en una mejora inmediata en la cognición para desarrollar una tarea particular y, ade- más, valorar cómo influye participar durante seis meses en uno de estos programas.

Aunque los resultados se analizarán más adelante, los investigadores coinciden en que constatan de forma subjetiva que las mejoras físicas y cognitivas son evidentes. "Muchos de los participantes llevaban una vida sedentaria y las mejoras son notables", explica Diego Pastor. Por su parte, Beatriz Bonete aprecia cómo el ejercicio físico mejora la memoria en los mayores.

Las mediciones de la parte llamada 'Neurogym', explica Bonete, se realizan antes y después de participar en el programa. Este test mide memoria a corto plazo, memoria visual inmediata, memoria visual diferida, concentración, cálculo y lógica. A lo largo del tiempo que dura 'Neurogym', los investigadores intervienen en todas las áreas para volver a medir los resultados al final. "Queremos comprobar cómo mejora la cognición después de un tiempo y, también, ver si se mantiene", aclara la investigadora.

\section{Participación social y optimismo}

Uno de los beneficios de participar en este tipo de iniciativas es el derivado de la cohesión grupal. La calidad de vida y la participación social del alumnado de los programas universitarios para mayores de la UMH han centrado la tesis doctoral de la investigadora de SABIEX Maica Segura. El trabajo analiza el proceso del envejecimiento desde una impacto de las necesidades de la población mayor y los recursos necesarios para crear el marco que garantice una buena calidad de vida y su integración activa en la sociedad. La investigadora destaca que los resultados concluyen que las personas mayores de 55 perspectiva psicosocial y tiene en cuenta el

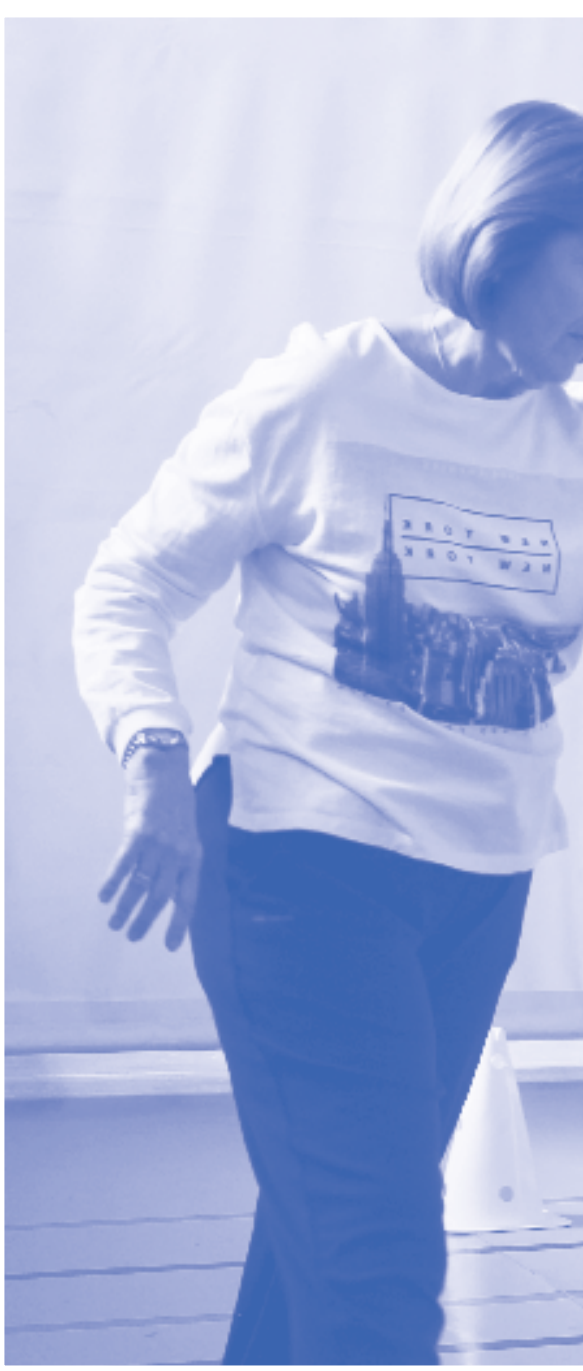

años con pareja tienen una percepción mejor de su calidad de vida global. Sin embargo, añade, se ha detectado que las personas del grupo calificadas como más jóvenes -menores de 65 años- que no tienen pareja, denotan tener una percepción mejor de su calidad de vida. Por otro lado, entre los sujetos con pareja, independientemente de su edad, quienes más participan socialmente y son más activos tienen una percepción mejor de su apoyo social. El trabajo de la profesora Segura destaca que el nivel de actividad e implicación social, dejando de lado los efectos negativos del envejecimiento, mejora la satisfacción vital.

Como la investigación se ha llevado a cabo con los inscritos en los programas universitarios para mayores de la $\mathrm{UMH}$, se ha concluido que estas iniciativas contribuyen a la mejora de su calidad de vida porque permiten la continuidad de una participación social y sirven para eliminar estereotipos negativos asociados a la edad ya que, además, están inmersos en un contexto intergeneracional. La profesora subraya que a través de estas iniciativas se puede evitar el aislamiento de las personas mayores y favorecer su bienestar en general. 


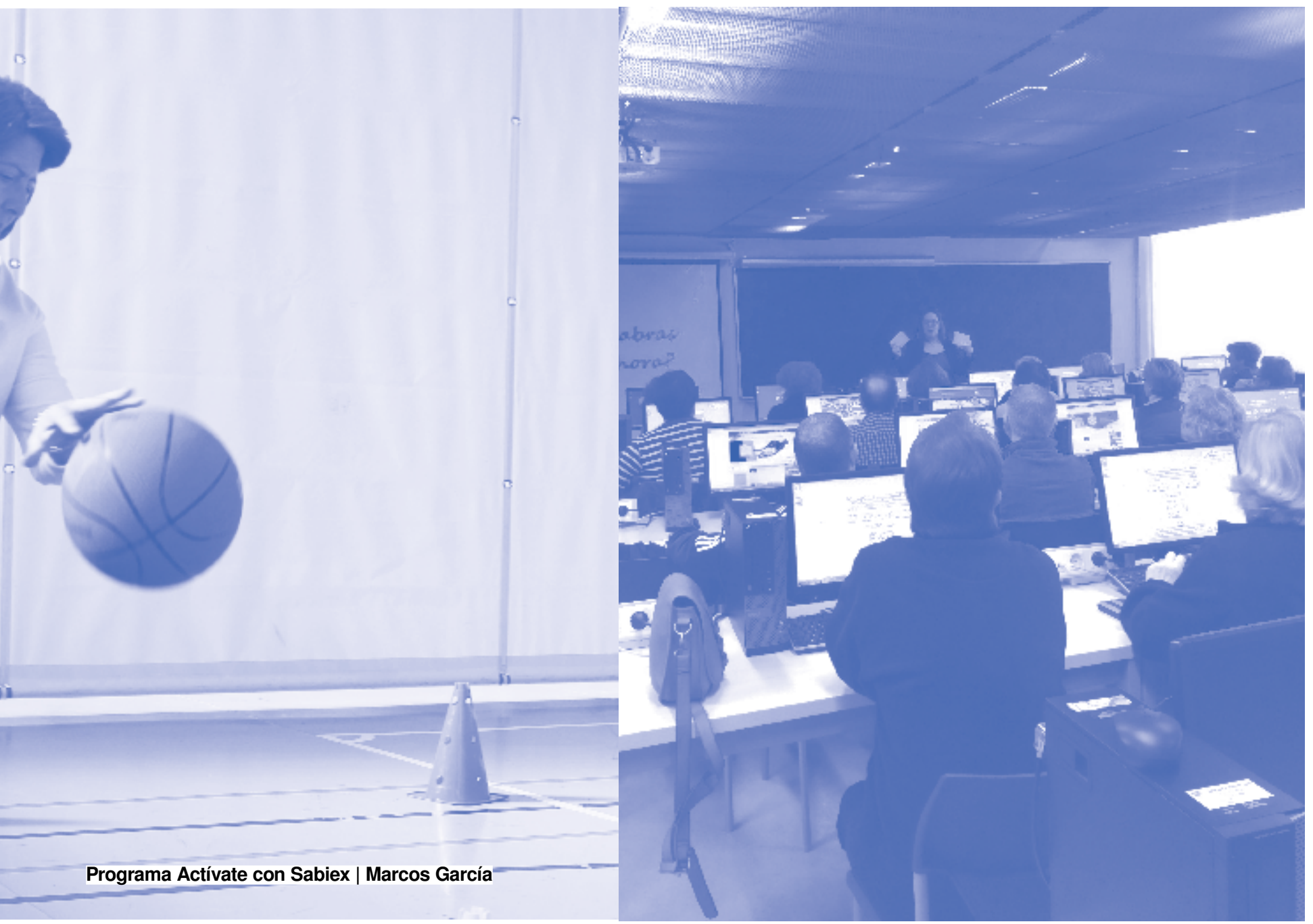

\section{Formación para acompañar}

La soledad de las personas mayores es un problema poco visible que requiere atención específica y soluciones concretas. La profesora Raquel Lorente trata de analizar las necesidades de los mayores que viven solos para ofrecer soluciones a la percepción subjetiva de la soledad. "La vejez suele asociarse a pérdida, pero también hay crecimiento y desarrollo", explica la investigadora. Lorente precisa que una de las cuestiones más visibles es que las personas que viven solas, aunque sea por decisión propia, acusan muchas veces soledad: "Pero no la declaran por temor a que se les institucionalice o se les prive de su independencia". El trabajo de la investigadora de SABIEX usa métodos mixtos para averiguar qué significado tiene la soledad para las personas en la provincia de Alicante y qué se hace para atender sus necesidades.

La investigación parte de la creación de un programa de formación de voluntarios llamado 'Acompaña-te' en el que miembros de la comunidad universitaria recibirán pautas específicas para llevar a cabo el acompañamiento. "Es necesaria una base sobre qué es el envejecimiento y las carac- terísticas que tiene esta etapa del desarrollo psicosocial para entenderla, empatizar, saber cómo se deben manejar las emociones propias y ser capaces de enseñar a otros a manejar las suyas", puntualiza Lorente. 'Acompaña-te' se fundamenta en los pilares de formación en envejecimiento y soledad, inteligencia emocional y voluntariado. "La soledad afecta a la salud de los mayores, por lo que es necesario aprender a gestionar este sentimiento", subraya la investigadora. Además de fundamentar el programa, el equipo analizará los beneficios que reporta tanto a la persona acompañada como al voluntario.

\section{Alfabetización y salud}

Las investigaciones sobre alfabetización en salud evidencian que, en función de la edad y la formación de la población, el consumo de recursos sanitarios cambia. Los mayores requieren más servicios de este tipo y un uso inadecuado conlleva un gasto económico mayor. La directora del grupo de investigación Calité, Virtudes Pérez, explica que una de sus investigaciones ha analizado el grado de alfabetización en salud en los participantes en los programas universitarios para mayores impulsados desde SABIEX. La cola-

\section{Las personas de edad avanzada son quienes más recursos sanitarios necesitan}

boración transversal ha permitido concluir que los integrantes de las Aulas de la Experiencia tienen mayor nivel de alfabetización los de otros estudios nacionales realizados con población mayor. Por ejemplo, explica la investigadora, saben cuándo deben acudir a su médico de atención primaria, en lugar de utilizar los servicios hospitalarios de urgencias. Virtudes Pérez cuenta que el siguiente paso es estudiar qué sucede en el caso de las personas mayores con menor nivel de alfabetización para proponer programas educativos específicos. "La formación a lo largo de la vida es beneficiosa en todos los aspectos", subraya la profesora de la UMH.

La investigación específica orientada a potenciar el bienestar de las personas mayores resulta imprescindible para mejorar su presente. El reto del programa SABIEX es conseguir que desde la Universidad se contribuya a conseguir que el envejecimiento sea un proceso activo y saludable. 